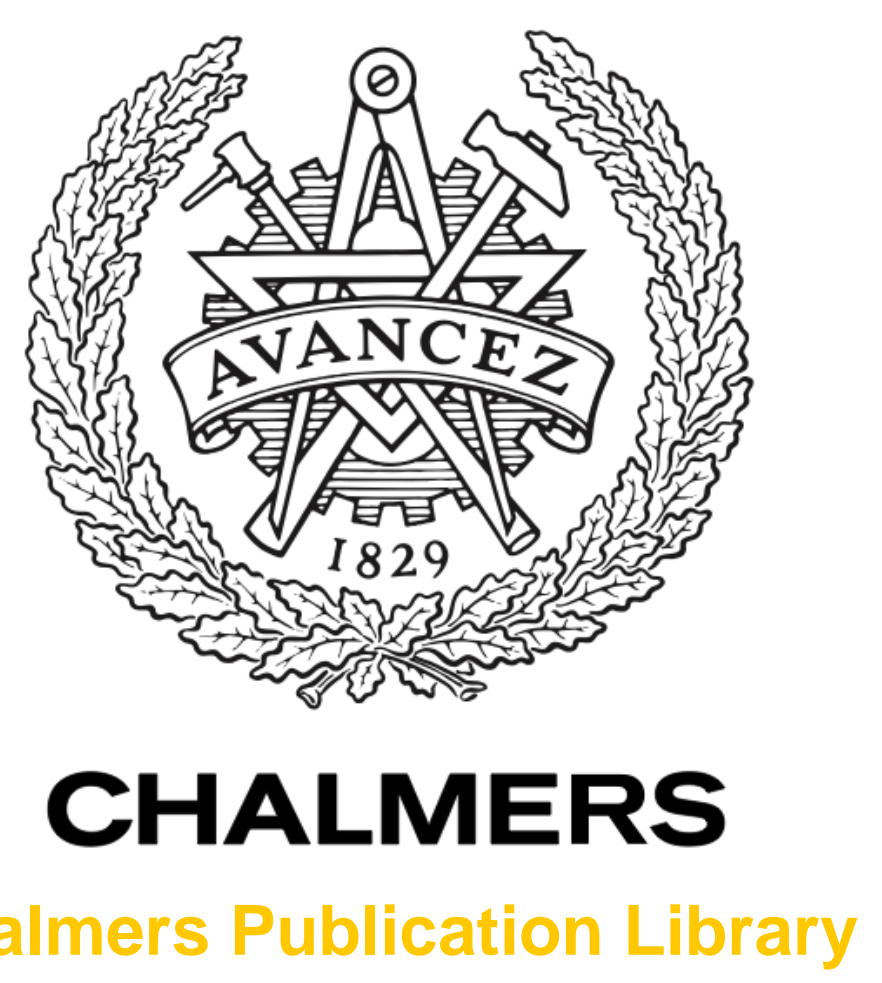

Chalmers Publication Library

\title{
A hybrid T matrix/boundary element method for elastic wave scattering from a defect near a non-planar surface
}

This document has been downloaded from Chalmers Publication Library (CPL). It is the author's version of a work that was accepted for publication in:

Journal of nondestructive evaluation (ISSN: 0195-9298)

Citation for the published paper:

Westlund, J. ; Boström, A. (2012) "A hybrid T matrix/boundary element method for elastic wave scattering from a defect near a non-planar surface". Journal of nondestructive

evaluation, vol. 31 pp. 148-156.

http://dx.doi.org/10.1007/s10921-012-0130-3

Downloaded from: http://publications.lib.chalmers.se/publication/168000

Notice: Changes introduced as a result of publishing processes such as copy-editing and formatting may not be reflected in this document. For a definitive version of this work, please refer to the published source. Please note that access to the published version might require a subscription.

Chalmers Publication Library (CPL) offers the possibility of retrieving research publications produced at Chalmers University of Technology. It covers all types of publications: articles, dissertations, licentiate theses, masters theses, conference papers, reports etc. Since 2006 it is the official tool for Chalmers official publication statistics. To ensure that Chalmers research results are disseminated as widely as possible, an Open Access Policy has been adopted.

The CPL service is administrated and maintained by Chalmers Library. 


\title{
A hybrid $T$ matrix/boundary element method for elastic wave scattering from a defect near a non-planar surface
}

\author{
J. Westlund, A. Boström \\ January 16, 2012
}

\begin{abstract}
The in-plane P-SV scattering of elastic waves by a defect and a close non-planar surface is considered. A hybrid $T$ matrix/boundary element approach is used, where a boundary integral equation is used for the non-planar surface and the Green's tensor in this integral equation is chosen as the one for the defect and thus incorporates the transition $(T)$ matrix of the defect. The integral equation is discretized by the boundary element method in a standard way. Also models of ultrasonic probes in transmission and reception are included. In the numerical examples the defect is for simplicity chosen as a circular cavity. This cavity is located close to a non-planar surface, which is planar except for a smooth transition between two planar parts. It is illustrated that the scattering by the cavity and the non-planar surface becomes quite complicated, and that shielding and masking may appear.
\end{abstract}

\section{Introduction}

The propagation and scattering of waves in elastic solids find important applications in ultrasonic testing and material characterization. Ultrasonic nondestructive testing is routinely used, e.g. in the nuclear power and aerospace industries, and ultrasound is of course also widely used in medical applications. The modelling of ultrasonic nondestructive testing is useful in many ways: it enhances the physical understanding, it makes it easy to perform parametric studies and thereby it is a useful tool in the development and qualification of testing procedures.

The propagation and scattering of waves in elastic solids have been studied extensively for a long time. However, not so many studies have been devoted to the development of complete models, including scattering by a defect, models of ultrasonic probes in transmission and reception, and calibration. Most complete models seem to depend on high frequency approximations (ray theory, geometrical theory of diffraction, and Kirchhoff theory), see e.g. Chapman [1], Calmon 
et al. [2] and Rose [3]. Boström and Wirdelius [4] give a model based on the $T$ matrix concept, in principle without any approximations, see e.g. Bövik and Boström [5] for similar work for cracks. However, this leads to other restrictions, such as only simply shaped defects and only planar component surfaces.

To be able to lift the restriction of planar surfaces, Westlund and Boström [6] give a boundary element method for the scattering by a straight crack close to a non-planar back surface, with the scattering by the crack treated by a hypersingular integral equation method. In the present paper another approach is explored in that a boundary element method for a non-planar back surface is coupled with a transition $(T)$ matrix representation of the defect. In principle any type of defect could be incorporated in this way, although the $T$ matrix of the defect must of course be computed in some way. One advantage with using the $T$ matrix is that it can be viewed as a 'black box', and can be easily replaced if the defect is changed. It is also straightforward to have a defect of another material, a situation that is somewhat more complicated to treat with a regular BEM method. For simplicity only a circular defect is used here in the numerical examples. To perform the coupling the Green's tensor used in the boundary integral equation for the back surface incorporates the scattering by the defect, and it is then natural to do this with the help of the $T$ matrix.

\section{Statement of the problem}

Consider the 2D in-plane scattering problem as depicted in Fig. 1. On the top (scanning) surface two ultrasonic probes, one transmitter $(\mathrm{T})$ and one receiver (R), are situated. The lower (back) surface may be of quite general shape, however it should have no cusps. The defect may be quite general, and it can be located close to the back surface. As the transition $(T)$ matrix will be used for the defect, a sufficient (but maybe not necessary) condition is that the circumscribed circle (with the chosen origin) of the defect does not intersect the back surface. As the multiple scattering between the scanning surface and the defect and back surface is neglected, the distance between these surfaces can not be too small (at least a couple of wavelengths). However, it should be stressed that the multiple scattering between the defect and the back surface is fully taken care of. The component is assumed to be homogeneous and isotropic with density $\rho$ and Lamé constants $\lambda$ and $\mu$.

Figure 1 also shows four collinear coordinate systems: one for the back surface (superscript 'b'), one for the defect ('d'), one for the transmitter ('t'), and one for the receiver ('r'). The position vectors relative to the back surface coordinate system for the transmitter and receiver systems and the defect system are $\boldsymbol{d}^{\mathrm{T}}, \boldsymbol{d}^{\mathrm{R}}$, and $\boldsymbol{d}^{\mathrm{D}}$, respectively. The shape of the back surface is determined by the equation $x_{2}^{\mathrm{b}}=g\left(x_{1}^{\mathrm{b}}\right)$. In the boundary element discretization of the back surface, it is truncated at the left and right truncation limits $T_{1}$ and $T_{2}$.

Time-harmonic conditions are assumed, and the factor $\mathrm{e}^{-\mathrm{i} \omega t}$, where $\omega$ is the angular frequency and $t$ the time, is suppressed throughout. The elastodynamic 


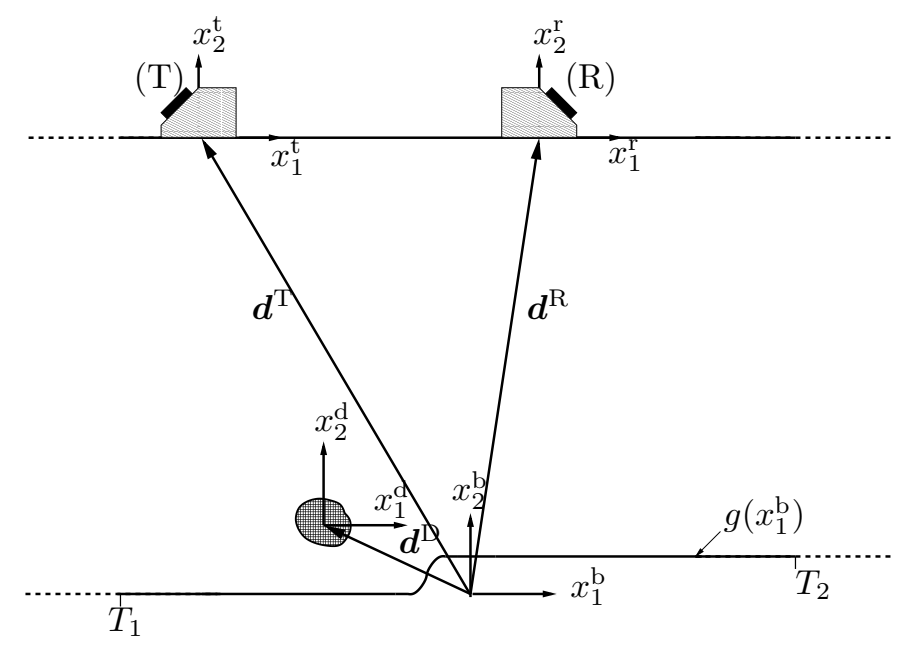

Figure 1: Illustration of the geometry with a defect in a thick-walled component with a non-planar back surface.

equation of motion is then:

$$
k_{\mathrm{p}}^{-2} \nabla(\nabla \cdot \boldsymbol{u})-k_{\mathrm{s}}^{-2} \nabla \times(\nabla \times \boldsymbol{u})+\boldsymbol{u}=\mathbf{0},
$$

where $k_{\mathrm{p}}=\omega / c_{\mathrm{p}}$ is the pressure wave number, $c_{\mathrm{p}}=\sqrt{(\lambda+2 \mu) / \rho}$ the pressure wave speed, $k_{\mathrm{s}}=\omega / c_{\mathrm{s}}$ the shear wave number, and $c_{\mathrm{s}}=\sqrt{\mu / \rho}$ the shear wave speed.

The boundary condition on the back surface is that it is free, i.e. the traction vanishes there. The scanning surface is also traction-free except for the action of the probes; the probes are treated in Sec. 5. The defect is not specified at this stage, it is only characterized through its $T$ matrix, it may be a cavity, a permeable scatterer, or a crack, in principle with any type of boundary conditions. To fully specify the problem radiation conditions must also be satisfied.

\section{The $T$ matrix and the Green's tensors}

The scattering by the defect and the back surface will be solved by a boundary integral equation approach. A direct method would be to use the outward propagating (free) Green's tensor and get two coupled boundary integral equations over the defect and the back surface. Here a different approach is taken in that the outward propagating Green's tensor for the defect is used. In this way the Green's tensor takes care of the boundary conditions on the defect and the boundary integral equation will be over the back surface only.

The Green's tensor for the defect can be constructed in different ways. Here it is simply assumed that the scattering properties of the defect are known in 
the form of the transition $(T)$ matrix of the defect. The $T$ matrix can in turn be computed in different ways; in the numerical examples the defect is taken as a circular cavity and then it is possible to calculate the $T$ matrix explicitly by separation-of-variables. Knowing the $T$ matrix it is a simple matter to determine the Green's tensor of the defect.

Introduce the cylindrical vector wave functions according to the definition:

$$
\begin{aligned}
& \chi_{1 m \sigma}(\boldsymbol{x})= \sqrt{\frac{\varepsilon_{m}}{4}}\left\{\hat{\boldsymbol{\rho}} \frac{m}{k_{\mathrm{s}} \rho} \mathrm{H}_{m}^{(1)}\left(k_{\mathrm{s}} \rho\right)\left(\begin{array}{c}
-\sin m \varphi \\
\cos m \varphi
\end{array}\right)-\hat{\boldsymbol{\varphi}} \mathrm{H}_{m}^{(1)^{\prime}}\left(k_{\mathrm{s}} \rho\right)\left(\begin{array}{c}
\cos m \varphi \\
\sin m \varphi
\end{array}\right)\right\} \\
& \chi_{2 m \sigma}(\boldsymbol{x})=\sqrt{\frac{\varepsilon_{m}}{4}} \frac{k_{\mathrm{p}}}{k_{\mathrm{s}}}\left\{\hat{\boldsymbol{\rho}} \mathrm{H}_{m}^{(1)^{\prime}}\left(k_{\mathrm{p}} \rho\right)\left(\begin{array}{c}
\cos m \varphi \\
\sin m \varphi
\end{array}\right)\right. \\
&\left.+\hat{\boldsymbol{\varphi}} \frac{m}{k_{\mathrm{p}} \rho} \mathrm{H}_{m}^{(1)}\left(k_{\mathrm{p}} \rho\right)\left(\begin{array}{c}
-\sin m \varphi \\
\cos m \varphi
\end{array}\right)\right\},
\end{aligned}
$$

where $(\rho, \varphi)$ are the polar coordinates of the position vector $\boldsymbol{x}$. Here, $\mathrm{H}_{m}^{(1)}$ is a Hankel function of the first kind, $\varepsilon_{m}$ is the Neumann factor (which may be expressed in terms of Kronecker's delta as: $\varepsilon_{m}=2-\delta_{m 0}$ ), and the convenient multiple index $n=j m \sigma$ is used where $j=1,2$ denotes the $\mathrm{SV}$-/P-wave function, respectively, $m=0,1,2, \ldots$ and $\sigma$ denotes even/odd parity (corresponding to the upper/lower equations). Also the regular counterparts $\operatorname{Re} \boldsymbol{\chi}_{n}$ to the wave functions, containing Bessel instead of Hankel functions, are used. Then any field incident on the defect (with sources outside the circumscribed circle of the defect) can be expanded in the regular wave functions as:

$$
\boldsymbol{u}^{i n, \mathrm{~d}}\left(\boldsymbol{x}^{\mathrm{d}}\right)=\sum_{n} a_{n} \operatorname{Re} \boldsymbol{\chi}_{n}\left(\boldsymbol{x}^{\mathrm{d}}\right),
$$

and the field scattered by the defect can be expanded as:

$$
\boldsymbol{u}^{s c, \mathrm{~d}}\left(\boldsymbol{x}^{\mathrm{d}}\right)=\sum_{n} f_{n} \boldsymbol{\chi}_{n}\left(\boldsymbol{x}^{\mathrm{d}}\right) .
$$

The last expansion is certainly valid outside the circumscribed circle of the defect, but may be valid also inside this circle (depending on the defect). The relation between the expansion coefficients $f_{n}$ and $a_{n}$ defines the $T$ matrix (expressed in the cylindrical basis):

$$
f_{n}=\sum_{n^{\prime}} T_{n, n^{\prime}} a_{n^{\prime}}
$$

The $T$ matrix may be computed by different methods, by separation-of-variables for a circular defect, by the null field approach for defects that are not too far from circular, and by the BEM or the FEM for more or less arbitrary defects.

The Green's displacement tensor for the defect $\boldsymbol{U}^{k}(\boldsymbol{x}, \boldsymbol{y} ; \omega)$, with corresponding stress tensor $\boldsymbol{\Sigma}^{k}(\boldsymbol{x}, \boldsymbol{y} ; \omega)$, satisfies the equation:

$$
\nabla \cdot \boldsymbol{\Sigma}^{k}(\boldsymbol{x}, \boldsymbol{y} ; \omega)+\rho \omega^{2} \boldsymbol{U}^{k}(\boldsymbol{x}, \boldsymbol{y} ; \omega)=-\delta(\boldsymbol{x}-\boldsymbol{y}) \mathbf{e}_{k}
$$


where $\delta(\boldsymbol{x}-\boldsymbol{y})$ is the 2D Dirac delta distribution and $\mathbf{e}_{k}$ the unit vector in the $k$-direction. The gradient operator is with respect to $\boldsymbol{x}$, which is thus the field point, whereas $\boldsymbol{y}$ is the source point. The Green's tensor also satisfies some boundary condition on the defect (and for a permeable defect also an equation inside the defect). The Green's tensor can naturally be decomposed into two terms: a free term and a defect term, such that $\boldsymbol{U}^{k}=\boldsymbol{U}^{k \text {,free }}+\boldsymbol{U}^{k \text {,defect }}$. The free term satisfies Eq. (3.5) in the whole plane and radiation conditions, and can be given in closed form in terms of Hankel functions. Alternatively, as long as $\left|\boldsymbol{x}^{\mathrm{d}}\right|<\left|\boldsymbol{y}^{\mathrm{d}}\right|$, the free term can be expanded in the cylindrical basis as:

$$
\boldsymbol{U}^{k, \text { free }}(\boldsymbol{x}, \boldsymbol{y} ; \omega)=\frac{\mathrm{i}}{\mu} \sum_{n} \operatorname{Re} \boldsymbol{\chi}_{n}\left(\boldsymbol{x}^{\mathrm{d}}\right) \boldsymbol{\chi}_{n}\left(\boldsymbol{y}^{\mathrm{d}}\right) \cdot \mathbf{e}_{k} .
$$

This is in the form of Eq. (3.2) with:

$$
a_{n}=\frac{\mathrm{i}}{\mu} \chi_{n}\left(\boldsymbol{y}^{\mathrm{d}}\right) \cdot \mathbf{e}_{k}
$$

This in turn means that the defect term of the Green's tensor can be written as:

$$
\boldsymbol{U}^{k, \operatorname{defect}}(\boldsymbol{x}, \boldsymbol{y} ; \omega)=\frac{\mathrm{i}}{\mu} \sum_{n, n^{\prime}} \chi_{n}\left(\boldsymbol{x}^{\mathrm{d}}\right) T_{n, n^{\prime}} \boldsymbol{\chi}_{n^{\prime}}\left(\boldsymbol{y}^{\mathrm{d}}\right) \cdot \mathbf{e}_{k}
$$

This expansion of the defect term is valid everywhere outside the circumscribed circle of the defect, where the term is also nonsingular.

\section{The integral equation and boundary element discretization}

The integral equation is derived using the $2 \mathrm{D}$ divergence theorem and a limiting process. In order to simplify the subsequent numerical computation of boundary element integrals, an indirect regularization approach is also followed. The derivation and regularization is completely analogous to the one in the paper by Westlund and Boström [6], so the details are omitted. The result is the following integral equation for the back surface displacements:

$$
\begin{aligned}
& -\int_{C_{B S}} u_{i}^{\mathrm{b}}\left(\boldsymbol{x}^{\mathrm{b}}\right)\left[\Sigma_{i j}^{k}\left(\boldsymbol{x}^{\mathrm{b}}, \boldsymbol{y}^{\mathrm{b}} ; \omega\right)-\Sigma_{i j}^{k}\left(\boldsymbol{x}^{\mathrm{b}}, \boldsymbol{y}^{\mathrm{b}}\right)\right] \mathrm{n}_{j}\left(\boldsymbol{x}^{\mathrm{b}}\right) \mathrm{d} s_{x}^{\mathrm{b}} \\
& -\int_{C_{B S}}\left[u_{i}^{\mathrm{b}}\left(\boldsymbol{x}^{\mathrm{b}}\right)-u_{i}^{\mathrm{b}}\left(\boldsymbol{y}^{\mathrm{b}}\right)\right] \Sigma_{i j}^{k}\left(\boldsymbol{x}^{\mathrm{b}}, \boldsymbol{y}^{\mathrm{b}}\right) \mathrm{n}_{j}^{\mathrm{b}}\left(\boldsymbol{x}^{\mathrm{b}}\right) \mathrm{d} s_{x}^{\mathrm{b}} \\
& -\frac{1}{2} u_{k}^{\mathrm{b}}\left(\boldsymbol{y}^{\mathrm{b}}\right)+u_{k}^{\mathrm{in}, \mathrm{b}}\left(\boldsymbol{y}^{\mathrm{b}}\right)=0, \quad k=1,2,
\end{aligned}
$$

where $C_{B S}$ is the truncated back surface (with lower and upper truncation limits $T_{1}$ and $T_{2}$, respectively), $\boldsymbol{y} \in C_{B S}, \boldsymbol{\Sigma}^{k}(\boldsymbol{x}, \boldsymbol{y})$ is the free static Green's tensor 
(i.e. the solution of Eq. (3.5) for $\omega=0)$, $\mathbf{n}$ is the downward unit normal vector of the back surface and $\boldsymbol{u}^{\text {in }}$ is the incident field from the transmitting probe. It should be noted that no defect term is present in Eq. (4.1) since the Green's tensor satisfies the boundary condition on the defect.

To solve the integral equation, the boundary element method (see e.g. Refs. [7, $8]$ ) is used with a standard isoparametric BE discretization of the back surface using quadratic Lagrangian interpolation functions. Letting $N_{E}$ denote the total number of boundary elements, the result of the discretization is a total of $2\left(2 N_{E}+1\right)$ unknown back surface node displacements. Collocating the integral equation at the node points generates the same number of equations, such that the integral equation is transformed into a system of $2\left(2 N_{E}+1\right) \times 2\left(2 N_{E}+1\right)$ linear algebraic equations.

\section{The probe model}

Conventional ultrasonic contact probes are considered in this paper, and the action of such probes in transmission can be modelled using the model developed by Boström and Wirdelius [4]. In this model the probe is assumed to be located on the surface of an elastic half-plane (i.e. multiple scattering between the probe and the back surface and defect is neglected), and the surface is free of tractions except beneath the probe where the traction is prescribed as:

$$
\boldsymbol{t}^{\mathrm{t}}= \begin{cases}A_{0} \mathrm{i} \mu k_{\mathrm{p}}\left[\delta \sin 2 \gamma_{\mathrm{t}} \mathbf{e}_{x_{1}^{\mathrm{t}}}+\left(\frac{k_{\mathrm{s}}^{2}}{k_{\mathrm{p}}^{2}}-2 \sin ^{2} \gamma_{\mathrm{t}}\right) \mathbf{e}_{x_{2}^{\mathrm{t}}}\right] \mathrm{e}^{-\mathrm{i} k_{\mathrm{p}} x_{1}^{\mathrm{t}} \sin \gamma_{\mathrm{t}}}, \mathrm{P} \text { probe } \\ A_{0} \mathrm{i} \mu k_{\mathrm{s}}\left[-\delta \cos 2 \gamma_{\mathrm{t}} \mathbf{e}_{x_{1}^{\mathrm{t}}}+\sin 2 \gamma_{\mathrm{t}} \mathbf{e}_{x_{2}^{\mathrm{t}}}\right] \mathrm{e}^{-\mathrm{i} k_{\mathrm{s}} x_{1}^{\mathrm{t}} \sin \gamma_{\mathrm{t}}}, & \text { SV probe }\end{cases}
$$

Here the probe angle $\gamma_{\mathrm{t}}$ is the rotation in the negative $x_{3}^{\mathrm{t}}$-direction as measured from the negative $x_{2}^{\mathrm{t}}$-axis, $A_{0}$ is an amplitude factor and the factor $\delta$ accounts for the effect of a couplant applied between the probe and scanning surface such that $\delta=0$ for fluid coupling and $\delta=1$ for a glued probe.

Prescribing the traction in this way is advantageous since the incident field from the transmitting probe may be analytically solved for in terms of a Fourier transform. Using the notation of Boström et al. [9] for the Fourier transform the incident field may be expressed as:

$$
\boldsymbol{u}^{\mathrm{in}, \mathrm{t}}\left(\boldsymbol{x}^{\mathrm{t}}\right)=\sum_{j=1}^{2} \int_{\Lambda_{-}} \xi_{j}(\beta) \boldsymbol{\varphi}_{j}^{\mathrm{t}}\left(\beta ; \boldsymbol{x}^{\mathrm{t}}\right) \mathrm{d} \beta,
$$

where the vector plane waves $\boldsymbol{\varphi}_{j}$ are defined by:

$$
\begin{aligned}
& \boldsymbol{\varphi}_{1}(\beta ; \boldsymbol{x})=-\frac{\mathrm{i} \hat{\boldsymbol{\beta}}}{8 \pi} \mathrm{e}^{\mathrm{i} k_{\mathrm{s}} \hat{\boldsymbol{\gamma}} \cdot \boldsymbol{x}}, \\
& \boldsymbol{\varphi}_{2}(\beta ; \boldsymbol{x})=\frac{k_{\mathrm{p}}}{k_{\mathrm{s}}} \frac{\mathrm{i} \hat{\boldsymbol{\gamma}}}{8 \pi} \mathrm{e}^{\mathrm{i} k_{\mathrm{p}} \hat{\boldsymbol{\gamma}} \cdot \boldsymbol{x}},
\end{aligned}
$$


the polar unit vectors are:

$$
\begin{aligned}
& \hat{\boldsymbol{\gamma}}=(\cos \beta, \sin \beta), \\
& \hat{\boldsymbol{\beta}}=(-\sin \beta, \cos \beta),
\end{aligned}
$$

and $\beta$ is the polar angle of propagation of the plane wave. It is thus seen that $\varphi_{1}$ is the $\mathrm{SV}$-wave and $\varphi_{2}$ the $\mathrm{P}$-wave. The integration contour $\Lambda_{-}$in the complex plane is defined by the directed segments $\beta \in(\pi+\mathrm{i} \infty, \pi) \cup[\pi, 2 \pi] \cup(2 \pi, 2 \pi-\mathrm{i} \infty)$, see Boström et al. [9] (where the contour is named $\Gamma_{-}$instead of $\Lambda_{-}$). The functions $\xi_{j}$ are determined by converting Eq. (5.2) to the rectangular coordinate $q$ in Fourier space, computing the corresponding traction and identifying with the Fourier transform $\boldsymbol{T}^{\mathrm{t}}$ of the prescribed traction $\boldsymbol{t}^{\mathrm{t}}$ given by Eq. (5.1). This yields:

$$
\begin{aligned}
& \xi_{1}(q)=\sqrt{\frac{2}{\pi}} \frac{h_{\mathrm{s}} k_{\mathrm{s}}}{\mu R}\left[\left(2 q^{2}-k_{\mathrm{s}}^{2}\right) T_{1}^{\mathrm{t}}-2 h_{\mathrm{p}} q T_{2}^{\mathrm{t}}\right], \\
& \xi_{2}(q)=\sqrt{\frac{2}{\pi}} \frac{h_{\mathrm{p}} k_{\mathrm{s}}}{\mu R}\left[2 h_{\mathrm{s}} q T_{1}^{\mathrm{t}}+\left(2 q^{2}-k_{\mathrm{s}}^{2}\right) T_{2}^{\mathrm{t}}\right],
\end{aligned}
$$

where $R=4 q^{2} h_{\mathrm{p}} h_{\mathrm{s}}+\left(2 q^{2}-k_{\mathrm{s}}^{2}\right)^{2}$ is the Rayleigh function and $h_{j}=h_{j}(q)=$ $\left(k_{j}^{2}-q^{2}\right)^{1 / 2}, j=\mathrm{p}$, s. The branch of the complex square root is chosen such that $\operatorname{Im} \sqrt{z} \geq 0 \forall z \in \mathbb{C}$. The Fourier transform $\boldsymbol{T}^{\mathrm{t}}$ of the prescribed traction $\boldsymbol{t}^{\mathrm{t}}$ is listed explicitly in the paper by Westlund and Boström [6]. The use of Eqs. (5.5) in Eq. (5.2) with $\xi_{1}(\beta)=\xi_{1}\left(q=k_{\mathrm{s}} \cos \beta\right), \xi_{2}(\beta)=\xi_{2}\left(q=k_{\mathrm{p}} \cos \beta\right)$ thus determines the incident field from the transmitting probe.

The expansion coefficients $a_{n}$ in the wave function expansion of the incident field, Eq. (3.2), must also be calculated and this is achieved by transforming the vector plane waves $\boldsymbol{\varphi}_{j}$ to the cylindrical vector wave functions $\operatorname{Re} \boldsymbol{\chi}_{n}$. This transformation is performed in two steps: the plane waves are first translated to an origin at the centre of the defect, and then expanded in the cylindrical wave functions. The details of these transformations are given in the chapter by Boström et al. [9], and applied to the present case one obtains:

$$
a_{j m \sigma}=\mathrm{i}^{m} \sqrt{\frac{\varepsilon_{m}}{2 \pi}} \int_{\Lambda_{-}} \xi_{j}(\beta) \mathrm{e}^{\mathrm{i} k_{j} \hat{\boldsymbol{\gamma}} \cdot \boldsymbol{d}^{\mathrm{TD}}}\left(\begin{array}{c}
\cos m \beta \\
\sin m \beta
\end{array}\right) \mathrm{d} \beta,
$$

where $\boldsymbol{d}^{\mathrm{TD}}=\boldsymbol{d}^{\mathrm{D}}-\boldsymbol{d}^{\mathrm{T}}$.

With the applications in mind being within ultrasonic nondestructive testing, it is of interest to compute the signal response obtained from the defect and the back surface. More explicitly the total signal response (i.e. with contributions both from the defect and the back surface) should be computed and compared to the signal response from the back surface only (i.e. with no defect present). For these computations Auld's reciprocity relation [10] is convenient to use, since it only presupposes knowledge of the action of the probe acting as transmitter. Auld's result relates two elastodynamic states: state (1) with displacement $\boldsymbol{u}^{(1)}$ and corresponding traction $\boldsymbol{t}^{(1)}=\boldsymbol{\sigma}^{(1)} \cdot \mathbf{n}$ obtained when the transmitting probe 
(T) acts as a transmitter in the presence of the scatterer, and state (2) with displacement $\boldsymbol{u}^{(2)}$ and corresponding traction $\boldsymbol{t}^{(2)}=\boldsymbol{\sigma}^{(2)} \cdot \mathbf{n}$ obtained when the receiving probe $(\mathrm{R})$ acts as a transmitter in the absence of the scatterer. The result is then that the change $\delta \Gamma$ in transmission coefficient (or reflection coefficient in the common case of pulse-echo testing where the same probe acts as both transmitter and receiver) due to the presence of the scatterer is given by:

$$
\delta \Gamma=\frac{\mathrm{i} \omega}{4 P} \int_{C}\left(\boldsymbol{u}^{(2)} \cdot \boldsymbol{t}^{(1)}-\boldsymbol{u}^{(1)} \cdot \boldsymbol{t}^{(2)}\right) \mathrm{d} s .
$$

The probes are here assumed to be transmitting at the angular frequency $\omega$. The contour $C$ is any contour enclosing the scatterer, $\mathbf{n}$ is the outward unit normal vector of the contour and the quantity $P$ is essentially the power supplied to the transmitting probe. The quantity $\delta \Gamma$ is directly proportional to the output voltage from the receiving probe, as discussed in the paper by Mattsson and Niklasson [11].

Auld's reciprocity relation is now used repeatedly to compute 1 ): the signal response due to the defect only, $\left.\delta \Gamma_{D}, 2\right)$ : the back surface signal response obtained with the defect present, $\delta \Gamma_{B S D}$, and 3): the back surface signal response obtained with the defect absent, $\delta \Gamma_{B S}$.

Starting with $\delta \Gamma_{D}$, this computation is based on Betti's identities for the cylindrical vector wave functions. These identities can be derived in the same way as for the spherical vector wave functions, see Pao [12], with the result that:

$$
\begin{gathered}
\int_{C}\left[\operatorname{Re} \boldsymbol{\chi}_{j^{\prime} m^{\prime} \sigma^{\prime}}(\boldsymbol{x}) \cdot \boldsymbol{t}\left(\operatorname{Re} \boldsymbol{\chi}_{j m \sigma}(\boldsymbol{x})\right)-\operatorname{Re} \boldsymbol{\chi}_{j m \sigma}(\boldsymbol{x}) \cdot \boldsymbol{t}\left(\operatorname{Re} \boldsymbol{\chi}_{j^{\prime} m^{\prime} \sigma^{\prime}}(\boldsymbol{x})\right)\right] \mathrm{d} s= \\
\int_{C}\left[\operatorname{Re} \boldsymbol{\chi}_{j^{\prime} m^{\prime} \sigma^{\prime}}(\boldsymbol{x}) \cdot \boldsymbol{t}\left(\boldsymbol{\chi}_{j m \sigma}(\boldsymbol{x})\right)-\boldsymbol{\chi}_{j m \sigma}(\boldsymbol{x}) \cdot \boldsymbol{t}\left(\operatorname{Re} \boldsymbol{\chi}_{j^{\prime} m^{\prime} \sigma^{\prime}}(\boldsymbol{x})\right)\right] \mathrm{d} s= \\
\mathrm{i} \mu \delta_{j j^{\prime}} \delta_{m m^{\prime}} \delta_{\sigma \sigma^{\prime}} .
\end{gathered}
$$

In these equations $\boldsymbol{t}$ denotes the traction operator (with outward unit normal vector) and $C$ is any contour of finite length enclosing the defect. Now let state (1) be with the defect present but the back surface absent and probe (T) generating the incident field, and state (2) be with both the defect and the back surface absent and probe $(\mathrm{R})$ generating the incident field. State (1) can then be represented as a sum of the wave function expansions given by Eqs. (3.2) and (3.3), while state (2) is simply the incident field and thus only requires the expansion (3.2). Both states are thus expanded in the wave functions such that the Betti identities may be used to explicitly calculate all integrals arising in the application of Auld's relation. The result is that the signal response due to the defect is given by:

$$
\delta \Gamma_{D}=\frac{\mu \omega}{4 P} \sum_{n, n^{\prime}} a_{n}^{(\mathrm{R})} T_{n, n^{\prime}} a_{n^{\prime}}^{(\mathrm{T})}
$$


where the expansion coefficients $a_{n}^{(\mathrm{R})}$ and $a_{n^{\prime}}^{(\mathrm{T})}$ of the incident fields from the probes (R) and (T), respectively, are calculated using Eq. (5.6).

Turning to the back surface signal response in the presence of the defect, $\delta \Gamma_{B S D}$, let state (1) be as above and state $(3)$ be with the defect and back surface present and probe (R) generating the incident field. An application of Auld's relation then gives:

$$
\delta \Gamma_{B S D}=\frac{\mathrm{i} \omega}{4 P} \int_{C_{B S}} u_{i}^{(3)}(\boldsymbol{x}) \sigma_{i j}^{(1)}(\boldsymbol{x}) \mathrm{n}_{j}(\boldsymbol{x}) \mathrm{d} s,
$$

where $\mathbf{n}$ is the downward unit normal vector of the back surface. The displacement $\boldsymbol{u}^{(3)}$ is computed by solving the BEM equations with probe (R) generating the incident field, and the stress $\boldsymbol{\sigma}^{(1)}$ is computed by applying the stress operator to the incident field given by Eq. (5.2) and the scattered field given by Eq. (3.3). It should be noted that the wave function expansion of the incident field, Eq. (3.2), should not be used here as it is only valid inside a circle, centered at the defect, not intersecting the probe $(\mathrm{T})$. For sufficiently large back surfaces this condition is not met, so the Fourier representation given by Eq. (5.2) must be used instead.

With $\delta \Gamma_{D}$ and $\delta \Gamma_{B S D}$ computed, the total signal response $\delta \Gamma_{T o t}$ is given by $\delta \Gamma_{T o t}=\delta \Gamma_{D}+\delta \Gamma_{B S D}$. This total signal response contains both the contributions due to direct scattering from the defect and back surface, and the response due to multiple scattering between the back surface and the defect.

Turning finally to the back surface response in the absence of the defect, $\delta \Gamma_{B S}$, let state (2) be as above and let state (4) be with the back surface present but the defect absent and probe $(\mathrm{T})$ generating the incident field. Auld's relation then yields:

$$
\delta \Gamma_{B S}=\frac{\mathrm{i} \omega}{4 P} \int_{C_{B S}} u_{i}^{(4)}(\boldsymbol{x}) \sigma_{i j}^{(2)}(\boldsymbol{x}) \mathrm{n}_{j}(\boldsymbol{x}) \mathrm{d} s .
$$

This signal response is useful for comparisons, as it gives the response of an unflawed medium. The displacement $\boldsymbol{u}^{(4)}$ is computed by solving the BEM equations with all element integrals computed using the free plane Green's tensor, i.e. without the additional defect term given by Eq. (3.8). The stress $\boldsymbol{\sigma}^{(2)}$ is simply the stress due to the incident field from the probe (R), and it is computed by applying Hooke's law to the corresponding Fourier representation given by Eq. (5.2).

\section{Numerical computations and results}

Turning to the numerical computations, the first step is the computation of the $T$ matrix. In the numerical examples presented in this section, the defect considered is a circular cavity. For this defect the $T$ matrix can be computed using the method of separation-of-variables [13]. This computation is straightforward and very efficient, and thus requires little comment. It should be noted, 


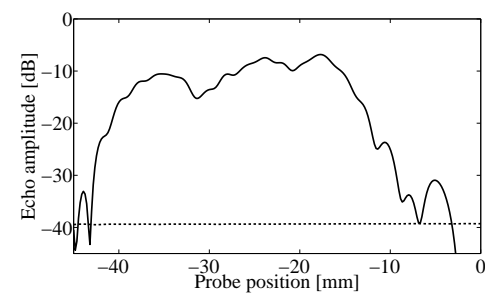

(a) Planar back surface.

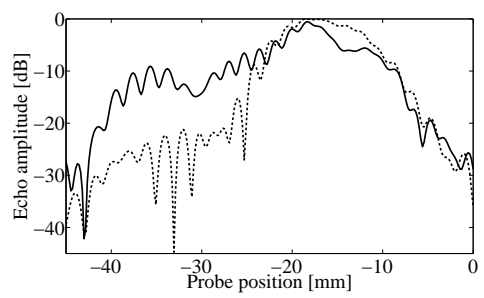

(b) Non-planar back surface.

Figure 2: The echo amplitude as a function of probe position, probe angle $\gamma_{\mathrm{t}}=-45^{\circ}$. Solid curves (-): defect present, dashed curves (- -): defect absent.

however, that for the case of no damping energy conservation considerations (see Ström [14]) lead to a useful check of the computation of the $T$ matrix: it must satisfy $T+T^{H}=-2 T^{H} T$, where the superscript $H$ denotes the Hermitian transpose. As to the required number of terms $m_{\max }$ in the wave function expansions, defect-only computations normally require very few terms, about $m_{\max }=k_{\mathrm{s}} b+6$ with $b$ denoting the radius of the defect. In this hybrid method, however, a larger number of terms may be required for accurate evaluation on the back surface of both the defect term (3.8) of the Green's tensor and the scattered part of the stress $\boldsymbol{\sigma}^{(1)}$. The actual number depends on the distance between the defect and the back surface, such that defects very close to the back surface require more terms. By numerical experiments it has proven sufficient to take $m_{\max }=k_{\mathrm{s}} b+6$ as long as the distance between the back surface and the defect is not less than $1 / 5$ shear wavelength. For smaller distances the number of terms must be increased, and a rule for assigning this number may readily be established based on numerical experiments. However, it should be noted that if the defect intersects the back surface then the solution method breaks down, since the defect term expansion given by Eq. (3.8) and the scattered wave field expansion given by Eq. (3.3) are then not valid.

Regarding the BE and incident wave field computations these do not require much special attention, except for the number of terms $m_{\max }$ required by the defect term (3.8) as noted above. These computations are otherwise analogous to those in the paper by Westlund and Boström [6], where further details can be found. One crucial point is the choice of the truncation limits $T_{1}$ and $T_{2}$ of the back surface. They are chosen so that the total discretized back surface length is roughly 100 shear wavelengths. The lengths of the boundary elements is about $1 / 4$ of the shear wavelength. These values are chosen so as to give good accuracy with negligible back scatter by the ends of the truncated back surface. That the back scatter from the ends is small can be checked easily in the time domain results, where the back scatter from the ends can be seen as small pulses at a known time. Alternatively, these pulses can be gated out in the time domain results (provided the frequency step is small enough, so that the time window is large enough). 


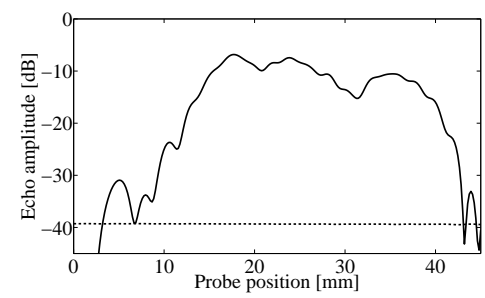

(a) Planar back surface.

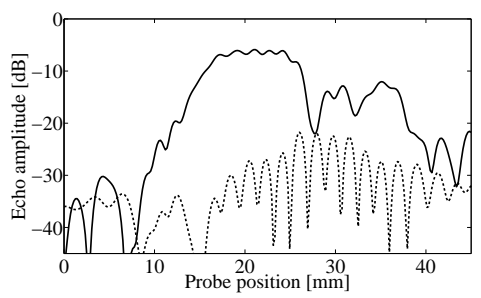

(b) Non-planar back surface.

Figure 3: The echo amplitude as a function of probe position, probe angle $\gamma_{\mathrm{t}}=45^{\circ}$. Solid curves (-): defect present, dashed curves (- -): defect absent.

Turning to numerical examples, these are for a pulse-echo testing situation such that the same probe is acting as both transmitter and receiver. The probe is taken as a fluid coupled (i.e. $\delta=0) 12 \mathrm{~mm} \mathrm{SV-probe} \mathrm{with} \mathrm{a} \mathrm{centre} \mathrm{frequency}$ of $1 \mathrm{MHz}$ and a frequency spectrum in the form of a Hanning window with a $6 \mathrm{~dB}$ bandwidth of $0.5 \mathrm{MHz}$. This means that a typical pulse length is a few microseconds, a fact that should be remembered when arrival times and overlap between pulses are discussed below. The probe is located at a vertical distance of $25 \mathrm{~mm}$ from the lowermost part of the back surface. The base material considered is steel with Lamé constants $\lambda=105 \mathrm{GPa}$ and $\mu=76 \mathrm{GPa}$ and density $\rho=7900 \mathrm{~kg} / \mathrm{m}^{3}$, such that the wave propagation speeds are $c_{\mathrm{p}} \approx 5.7 \mathrm{~mm} / \mu \mathrm{s}$ and $c_{\mathrm{s}} \approx 3.1 \mathrm{~mm} / \mu \mathrm{s}$. Material damping is incorporated in the model by giving the Lamé constants imaginary parts of $1 \%$ of the real parts. The defect is a circular cavity of radius $2 \mathrm{~mm}$ located at $\boldsymbol{d}^{\mathrm{D}}=\{0,6\} \mathrm{mm}$. The back surface is either planar or with a transition in the form of a quarter-wavelength sine function from $x_{2}^{\mathrm{b}}=0$ to $x_{2}^{\mathrm{b}}=4 \mathrm{~mm}$ over the interval from $x_{1}^{\mathrm{b}}=4 \mathrm{~mm}$ to $x_{1}^{\mathrm{b}}=10 \mathrm{~mm}$. This back surface is chosen as a simple illustration of the effect of the back surface geometry, but it should be remembered that in principle any back surface geometry can be handled since the BEM is used for the back surface.

Figure 2 shows the pulse-echo signal response as a function of probe position, computed for the centre frequency and a probe angled $45^{\circ}$ to the right, i.e. $\gamma_{\mathrm{t}}=-45^{\circ}$. The dashed lines give the back surface signal response in the absence of the defect, while the full-drawn lines show the total signal response with the defect present. Figure 3 shows the corresponding results for a probe instead angled $45^{\circ}$ to the left, i.e. $\gamma_{t}=45^{\circ}$.

In Figs. 2(a) and 3(a) the results for the planar back surfaces are given, while Figs. 2(b) and 3(b) give the results for the non-planar. The results are uncalibrated but the same normalization is used such that they can be compared. Since the probes are angled the planar back surface gives only very weak signal responses in Figs. 2(a) and 3(a), but the responses from the defect are quite strong with peaks from the "corner" (i.e. hitting both the back surface and the defect) and direct echoes. In Fig. 2(b) on the other hand, the non-planar part of the back surface gives a very strong response. Additionally, with the defect 


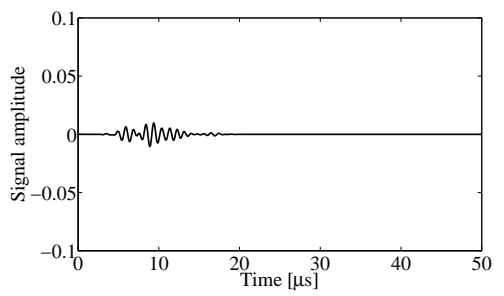

(a) Planar back surface, defect absent.

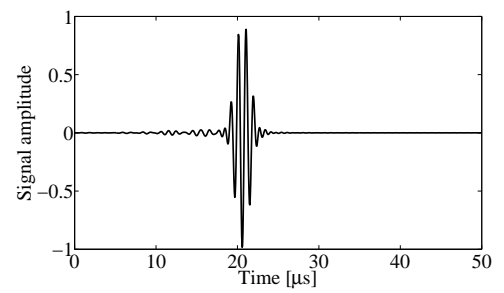

(c) Non-planar back surface, defect absent.

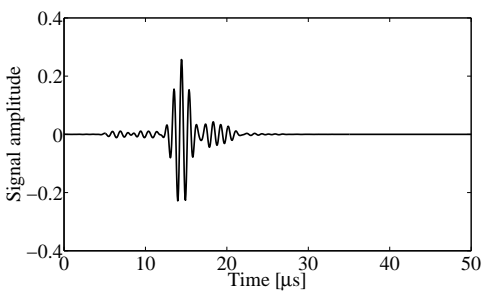

(b) Planar back surface, defect present.

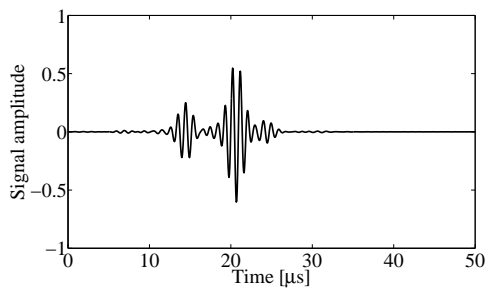

(d) Non-planar back surface, defect present.

Figure 4: The time traces for a probe located $15.4 \mathrm{~mm}$ to the left of the defect, probe angle $\gamma_{\mathrm{t}}=-45^{\circ}$.

present this part of the back surface is partly shielded such that the total signal response is weaker than without the defect. Finally it may also be noted in Fig. 3(b) that, as expected, the defect gives a response which is much easier to distinguish from the back surface response when the probe is angled to the left instead.

Figures 4 and 5 show the time traces for probe positions at $15.4 \mathrm{~mm}$ and $8.2 \mathrm{~mm}$ to the left of the defect centre, respectively, for the probe angled $45^{\circ}$ to the right. The normalization is the same in all the time traces, but it should be noted that different scales are used.

In Fig. 4(a), only the wave pulses travelling (almost) vertically down to the back surface and back up to the probe again are present. The pure P-wave pulse has an arrival time of $t \approx 8.7 \mu \mathrm{s}$, but the probe edge contributions appearing at $t \approx 6.0 \mu$ s and $t \approx 11.5 \mu$ s are also clearly visible. The latter, however, overlaps the mode-converted pulse appearing at $t \approx 12.4 \mu \mathrm{s}$. Finally, the pure $\mathrm{SV}$-wave pulse with an arrival time of $t \approx 16.1 \mu$ s can also be identified in the trace. With the defect present, Fig. 4(b), the direct echo from the pure SV-wave pulse with an arrival time of $t \approx 14.5 \mu$ s dominates, but the direct echoes from the pure $\mathrm{P}$-wave and mode converted pulses with arrival times of $t \approx 7.9 \mu \mathrm{s}$ and $t \approx 11.2 \mu \mathrm{s}$, respectively, are also visible. The defect signal response also reveals a pulse appearing at $t \approx 18.0 \mu$ s and this is a creeping wave: the SV-wave hits the defect and generates a Rayleigh-like surface wave which travels around the back of the defect and returns to the probe as an SV-wave. In the total signal 


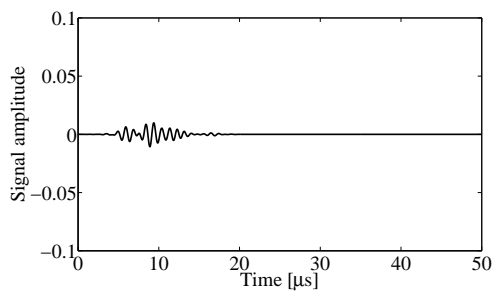

(a) Planar back surface, defect absent.

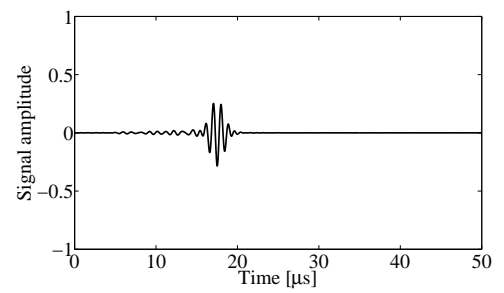

(c) Non-planar back surface, defect absent.

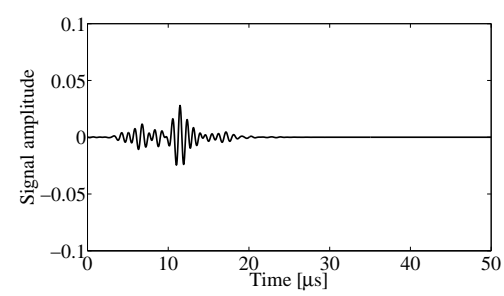

(b) Planar back surface, defect present.

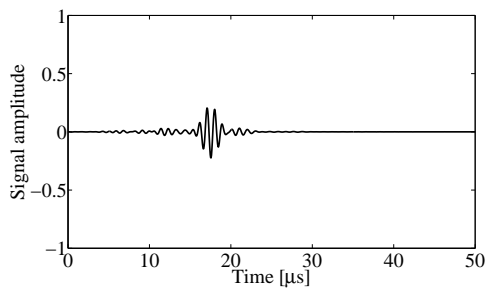

(d) Non-planar back surface, defect present.

Figure 5: The time traces for a probe located $8.2 \mathrm{~mm}$ to the left of the defect, probe angle $\gamma_{\mathrm{t}}=-45^{\circ}$.

response shown in Fig. 4(b), this pulse is overlapped by the quite strong pure $\mathrm{SV}$-wave corner echo pulse with an arrival time $t \approx 18.6 \mu \mathrm{s}$.

The time trace for the non-planar back surface with defect absent, Fig. 4(c), features the same pulses as in Fig. 4(a) but in addition there are also the direct echoes from the non-planar part of the back surface with arrival times $t \approx$ $11.2 \mu \mathrm{s}, t \approx 16.0 \mu \mathrm{s}$ and $t \approx 20.7 \mu \mathrm{s}$ for the pure $\mathrm{P}$-wave, mode converted and pure SV-wave pulses, respectively. Since the probe is an SV-probe, the latter pulse dominates completely. With the defect present, Fig. 4(d), the same defect pulses as in Fig. 4(b) and back surface pulses as in Fig. 4(c) are present, although the latter are weaker with the defect present due to a shielding effect. However, quite strong pulses due to multiple scattering between the back surface and defect are now also possible. A pure SV-wave hitting the non-planar part of the back surface, scattered by the defect back to the non-planar surface and finally reaching the probe again has a wave path length corresponding to an arrival time of $t \approx 24.6 \mu \mathrm{s}$ and this pulse is clearly visible in Fig. 4(d). The possibilities of multiple scattering between the defect and back surface also gives rise to weak pulses arriving even later in the time trace.

Many of the pulses in Fig. 5 are naturally the same as those in Fig. 4, and the other can be identified in an analogous analysis. The main difference is that the shielding effect of the defect on the back surface signal response is much smaller for this probe position, so the back surface echoes are nearly identical in Figs. 5(c) and 5(d). 


\section{Concluding remarks}

In the present paper a model of ultrasonic testing for defects near a back surface of general, complex geometry is developed. The defect is represented by its $T$ matrix, and may thus in principle be arbitrary. The wave scattering problem is solved in the frequency domain using the boundary element method, with the Green's tensor for the defect in the kernel, such that integrals over the defect are avoided. The incident field from an ultrasonic contact probe in transmission is calculated in terms of an inverse Fourier transform, in a probe model based on prescribing the traction on the scanning surface. The action of the receiving probe is modelled using an electromechanical reciprocity argument, and an inverse temporal Fourier transform is applied to compute the time traces.

The 2D in-plane wave propagation is treated in the paper, but the model could readily be extended to treat the 3D case for which a library of computed $T$ matrices for different defects, such as circular cracks and volumetric defects, exists.

\section{Acknowledgments}

The present work is sponsored by the Swedish Radiation Safety Authority, SSM, and this is gratefully acknowledged.

\section{References}

[1] R.K. Chapman. A system model for the ultrasonic inspection of smooth planar cracks. J. Nondestr. Eval., 9:197-210, 1990.

[2] P. Calmon, A. Lhémery, I. Lecæur-Taïbi, R. Raillon, and L. Paradis. Models for the computation of ultrasonic fields and their interaction with defects in realistic NDT configurations. Nucl. Eng. Des., 180:271-283, 1998.

[3] J.L. Rose. Ultrasonic Waves in Solid Media. Cambridge University Press, Cambridge, 1999.

[4] A. Boström and H. Wirdelius. Ultrasonic probe modeling and nondestructive crack detection. J. Acoust. Soc. Am., 97:2836-2848, 1995.

[5] P. Bövik and A. Boström. A model of ultrasonic nondestructive testing for internal and subsurface cracks. J. Acoust. Soc. Am., 102:2723-2733, 1997.

[6] J. Westlund and A. Boström. A 2D model of ultrasonic testing for cracks near a non-planar surface. Wave Motion, 47:383-394, 2010.

[7] M. Bonnet. Boundary Integral Equation Methods for Solids and Fluids. John Wiley \& Sons Ltd, Chichester, 1995.

[8] J. Domínguez. Boundary Elements in Dynamics. Computational Mechanics Publications, Southampton, 1993. 
[9] A. Boström, G. Kristensson, and S. Ström. Transformation properties of plane, spherical and cylindrical scalar and vector wave functions. In Varadan et al. [15].

[10] B.A. Auld. General electromechanical reciprocity relations applied to the calculation of elastic wave scattering coefficients. Wave Motion, 1:3-10, 1979 .

[11] J. Mattsson and A. J. Niklasson. Ultrasonic 2-D SH crack detection in anisotropic solids. J. Nondestr. Eval., 16:31-41, 1997.

[12] Y-H. Pao. Betti's identity and transition matrix for elastic waves. J. Acoust. Soc. Am., 64:302-310, 1978.

[13] V.V. Varadan, Y. Ma, V.K. Varadan, and A. Lakhtakia. Scattering of waves by spheres and cylinders. In Varadan et al. [15].

[14] S. Ström. The scattered field. In Varadan et al. [15].

[15] V.V. Varadan, A. Lakhtakia, and V.K. Varadan, editors. Field Representations and Introduction to Scattering, volume 1 of Acoustic, electromagnetic and elastic wave scattering. North-Holland, Amsterdam, 1991. 